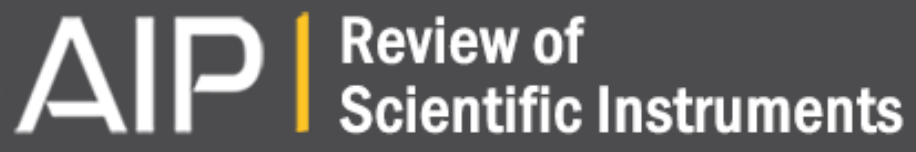

SODI-COLLOID: A combination of static and dynamic light scattering on board the International Space Station

S. Mazzoni, M. A. C. Potenza, M. D. Alaimo, S. J. Veen, M. Dielissen, E. Leussink, J.-L. Dewandel, O. Minster,

E. Kufner, G. Wegdam, and P. Schall

Citation: Review of Scientific Instruments 84, 043704 (2013); doi: 10.1063/1.4801852

View online: http://dx.doi.org/10.1063/1.4801852

View Table of Contents: http://scitation.aip.org/content/aip/journal/rsi/84/4?ver=pdfcov

Published by the AIP Publishing

\section{Articles you may be interested in}

A novel full-angle scanning light scattering profiler to quantitatively evaluate forward and backward light scattering from intraocular lenses

Rev. Sci. Instrum. 86, 095004 (2015); 10.1063/1.4930179

Multi-function Light Microscopy Module for the International Space Station

AIP Conf. Proc. 504, 324 (2000); 10.1063/1.1302498

Determination of hydrodynamic properties in highly charged colloidal systems using static and dynamic light scattering

J. Chem. Phys. 110, 7070 (1999); 10.1063/1.478612

Combustion studies aboard the international space station: Planned experiments and facilities

AIP Conf. Proc. 458, 82 (1999); 10.1063/1.57660

Surface defect scattering of surface plasmon polaritons: Mirrors and light emitters

Appl. Phys. Lett. 73, 3509 (1998); 10.1063/1.122820
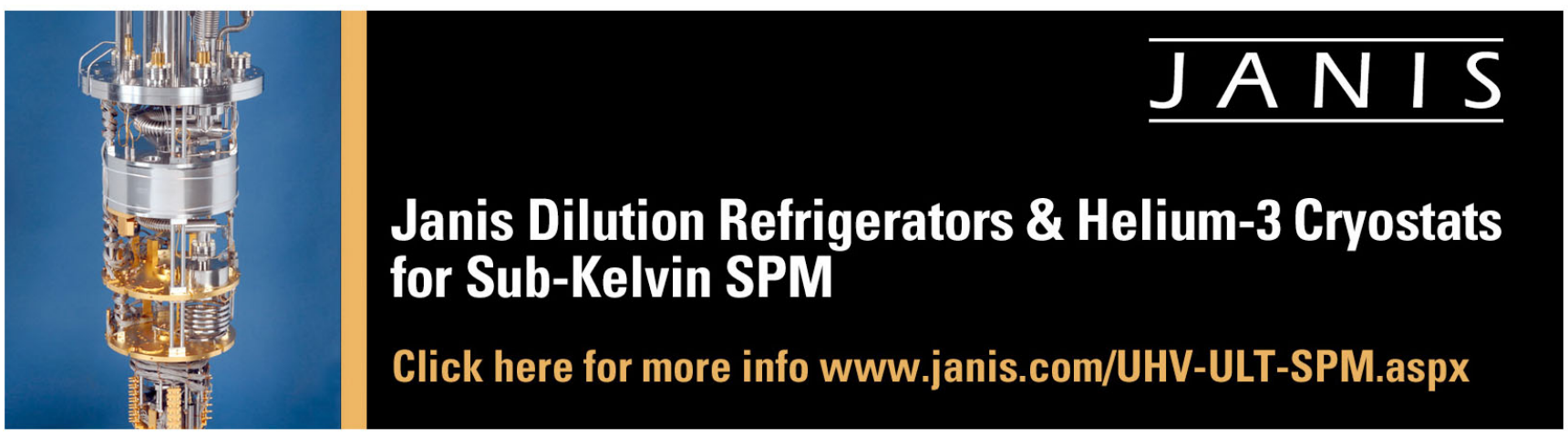


\title{
SODI-COLLOID: A combination of static and dynamic light scattering on board the International Space Station
}

\author{
S. Mazzoni, ${ }^{1, a), b)}$ M. A. C. Potenza, ${ }^{2}$ M. D. Alaimo, ${ }^{2}$ S. J. Veen, ${ }^{3, c}$ M. Dielissen, ${ }^{4}$ \\ E. Leussink, ${ }^{4}$ J.-L. Dewandel, ${ }^{5}$ O. Minster, ${ }^{1}$ E. Kufner, ${ }^{1}$ G. Wegdam, ${ }^{3}$ and P. Schall ${ }^{3}$ \\ ${ }^{1}$ Human Spaceflight Directorate, European Space Agency, Keplerlaan 1, $2200 A$ G Noordwijk, The Netherlands \\ ${ }^{2}$ Dipartimento di Fisica, Università degli Studi di Milano, via Celoria 16, I-20133 Milano, Italy \\ ${ }^{3}$ van der Waals-Zeeman Institute, University of Amsterdam, Valckenierstraat 65, 1018XE Amsterdam, \\ The Netherlands \\ ${ }^{4}$ QinetiQ Space nv, Hogenakkerhoekstraat 9, 9150 Kruibeke, Belgium \\ ${ }^{5}$ Lambda-X sa, Rue de l'Industrie 37, 1400 Nivelles, Belgium
}

(Received 11 January 2013; accepted 1 April 2013; published online 22 April 2013)

\begin{abstract}
Microgravity research in space is a complex activity where the often scarce resources available for the launch, accommodation, and operation of instrumentation call for a careful experiment planning and instrument development. In this paper we describe a module of the Selectable Optical Diagnostic Instrument, that has been designed as a compact optical diagnostic instrument for colloidal physics experiments. The peculiarity of the instrument is the combination of a novel light scattering technique known as near field scattering and standard microscopy with a low-coherence laser light source. We describe its main design features, as well as measurement results on colloidal aggregation taken on the International Space Station. ( 2013 AIP Publishing LLC. [http://dx.doi.org/10.1063/1.4801852]
\end{abstract}

\section{INTRODUCTION}

Scientific experiments performed in microgravity require specially designed instrumentation and measurement equipment because of the very stringent conditions dictated by the space environment. At present, a large portion of reduced gravity experiments are performed on board the International Space Station (ISS), a permanently inhabited satellite orbiting around the earth at an altitude range of $300-400 \mathrm{~km}$ that hosts laboratories for physics, biology, and human physiology experiments. ${ }^{1}$ The instrumentation used there needs to attain the highest level of reliability because any servicing capabilities, if available, are extremely limited once the instrument has been launched in space. The design must be rugged enough to assure a constant performance based on the calibration on ground, and it must be able to survive the launch, a brief (e.g., minutes) period of very intense vibration and acceleration that can reach $4 \mathrm{~g}$ in the Russian "Soyuz" rocket, routinely used for microgravity science missions. In the case of optical instruments, this means guaranteeing robust optical alignment within a fraction of a micrometer.

In this paper, we will focus our attention on the Near Field Scattering (NFS) unit of the Selectable Optical Diagnostic Instrument (SODI). Throughout the paper, we will mention the instrument with its space mission name: SODICOLLOID. The instrument was developed by an industrial consortium under contract to the European Space Agency to carry out selected physics experiments in the field of soft matter.

\footnotetext{
a) Present address: CERN CH-1211, Geneva 23, Switzerland.

b) Author to whom correspondence should be addressed. Electronic mail: stefano.mazzoni@cern.ch

c) Present address: Unilever R\&D Vlaardingen, Olivier van Noortlaan 120, 3122 AT Vlaardingen, The Netherlands.
}

Its development started in 2005 following the approval of a series of scientific experiments on various colloidal systems, including short-range attractive colloidal systems. ${ }^{2}$ It was launched to the ISS in 2010. The typical configuration of such a "multi-user" instrument sees a series of instrument specific inserts (typically the cell) that can be easily integrated into a fixed, general instrument. The investment incurred in the development and launch of space-grade instrumentation is generally so high that utilisation needs to be maximised by performing experiments with similar experimental needs in the same facility. Launch and development costs are therefore limited to the elements that can be removed and exchanged by astronauts once the facility is installed. A key element of early development was the choice of a technique that could be executed easily from ground with minimum or no intervention from the astronauts. Despite the increase of the number of crew members on board the spacecraft in 2009, the availability of crew time remains scarce. As a result, manual intervention is limited to the installation/de-installation of the instrument and, in case of off-nominal performance, of very basic operations as visual inspection, mating/de-mating of connectors, etc. The most common problems of optical instruments (misalignment above others) can therefore be fatal since corrective measures cannot be performed on board the spacecraft.

These considerations resulted in the choice of NFS, a heterodyne, self-referencing optical technique of recent development with characteristics that make it appealing for space experiments, being extremely simple in its layout, almost entirely alignment-free, and with relaxed requirements for the quality of optical components. Moreover, NFS allows simultaneous measurement of static and dynamic light scattering (DLS) by imaging the near field scattered light. As such, it is ideally suited for following structural growth processes on the colloidal length scale. In this paper, we present 
measurement results of the aggregation of colloidal particles interacting via critical Casimir forces. Because of the attractive critical Casimir interactions, the particles aggregate into fractal structures. Microgravity conditions are essential to observe the diffusion-limited growth of these aggregates without disturbance by convection and sedimentation, as shown by comparative measurements in microgravity and on ground. ${ }^{11}$ The control of the critical Casimir interactions needs precise temperature control; additionally, stirring is required in between measurements to resuspend the particles. All these performances are incorporated in the fully automated SODICOLLOID instrument. The paper will be organised as follows: we will provide a description of the NFS technique in Sec. II, a description of the instrument in Sec. III, a description of the sample in Sec. IV, and the data analysis and experimental results from the space mission in Secs. V and VI. The calibration procedure is described in detail in Appendixes A-C.

\section{NFS MEASUREMENT TECHNIQUE}

NFS is a light scattering technique that allows the measurement of the density correlation function of a sample. ${ }^{3-5}$ In contrast to usual far-field light scattering measurements, in NFS, light scattered by the sample is recorded at a close distance, together with the transmitted light beam (heterodyne configuration). The advantage is that a large range of scattering angles can be recorded simultaneously. Hence, compared to regular light scattering, NFS allows simultaneous time- and space resolved measurements. The NFS optical layout substantially follows what is described in Ref. 5 and references therein (see Fig. 1).

The laser beam, launched via an optical fibre, illuminates the sample after being collimated by a lens. The illuminated region has a Gaussian transverse profile with an intensity distribution larger than the cell, so that the entire field of view (FOV) is almost uniformly illuminated, the maximum intensity variation being smaller than a factor 2 with respect to the maximum value. The superposition of transmitted and scattered light is projected onto the detector using a microscope objective. The detector plane is conjugate to the object plane, situated at a distance $\mathrm{z}$ just behind the sample, so that an image (from now on referred as "NFS image") of the near-field scattered light is produced (see Fig. 1). Sequences of NFS images are finally recorded on a camera and stored on a computer for image analysis.

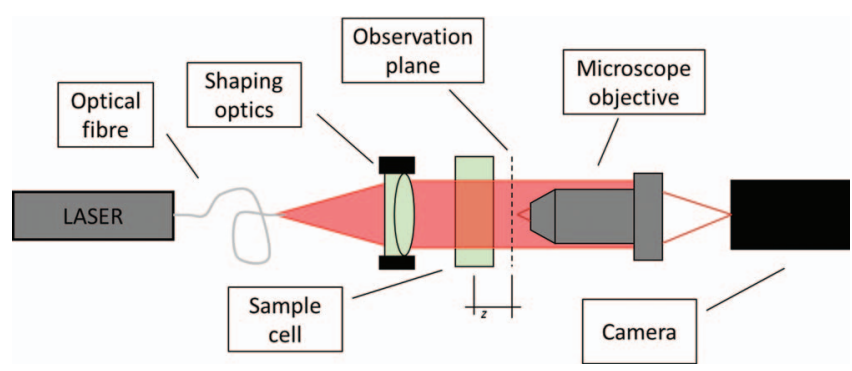

FIG. 1. Schematic of the NFS setup used for the COLLOID experiment.

\section{THE SODI-COLLOID MODULE}

NFS was selected by the European Space Agency as the technique of choice for the SODI-COLLOID module. The instrument has been designed to meet the stringent engineering budgets of space payloads. At variance with the ground laboratory version of NFS, in the space version, the laser beam is deflected by optical mirrors (see Fig. 2) allowing the instrument to fit a container of size $314 \times 622 \times 228 \mathrm{~mm}^{3}$ and reaching a total mass of $21 \mathrm{~kg}$ (including the container). The setup is composed as follows. The light source (1) is a commercial Distributed Feedback (DFB) laser diode emitting at $935 \mathrm{~nm}$, coupled to a single mode optical fibre. The DFB laser diode was sinusoidally modulated in current at $20 \mathrm{kHz}$ to decrease its apparent coherence length to a few mm's and alleviate high contrast interference fringes occurring from multiple reflections between the cell and the objective front lens. The beam is collimated (2) resulting in a beam size of an approximate diameter of $10 \mathrm{~mm}\left(\mathrm{dia} 1 / \mathrm{e}^{2}\right)$. After being deflected by the first mirror (3), the beam impinges on the sample cell hosted in an array of 5 cells. The cell array is mounted on a computer controlled translation stage (4) that selects the active cell by positioning it in the optical path. The cell is imaged by a long $(17 \mathrm{~mm})$ working distance $20 \times$ magnification microscope objective (5) with NA $=0.3$ and multilayer coating for near-infrared radiation. It can be moved in such a way that the focus lies inside the sample, thereby providing realspace images of the central plane of the sample. We will refer to this position as the imaging position, placed at $\mathrm{z}=0$. To acquire NFS images, however, the lens was moved to a distance $\mathrm{z}=2.9 \mathrm{~mm}$ behind the sample. The image of the objective lens is then projected onto a 10-bit CCD $1024 \times 1024$ sensor (7) using a lens with a focal length of $200 \mathrm{~mm}$ (6). This design covers a range of wave vectors from approximately $\mathrm{q}_{\mathrm{min}}$ $=0.03 \mu \mathrm{m}^{-1}$ up to $\mathrm{q}_{\max }=1.9 \mu \mathrm{m}^{-1}$.

The sample cells are $25 \times 10 \times 2(\mathrm{H} \times \mathrm{W} \times \mathrm{D})$ $\mathrm{mm}^{3}$ optical grade quartz cuvettes sealed by a double o-ring

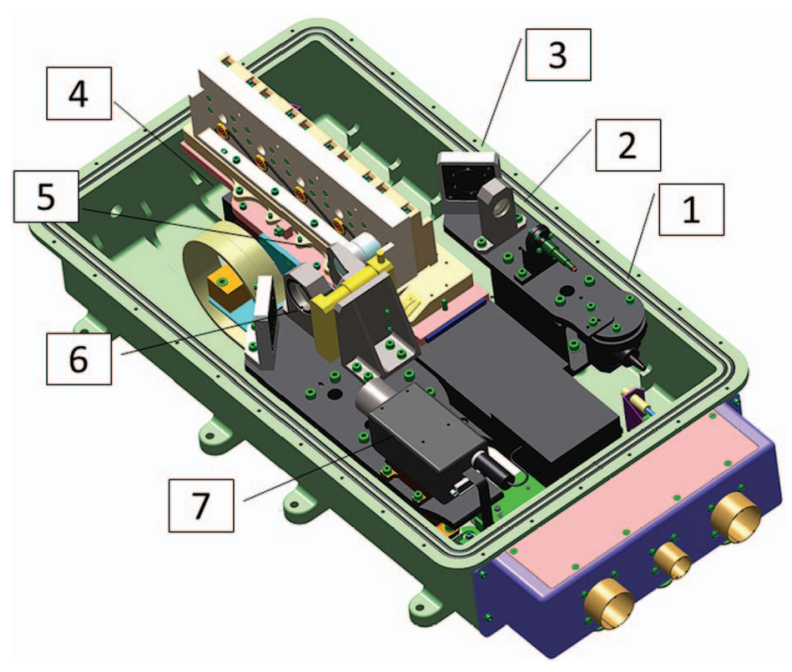

FIG. 2. Drawing of the SODI-COLLOID module. A movable array of cells is mounted in front of the collection optics to allow measurement of multiple samples. Due to safety regulations for instruments that are operated on board the ISS, the module is enclosed in a sealed container that cannot be opened in orbit. 


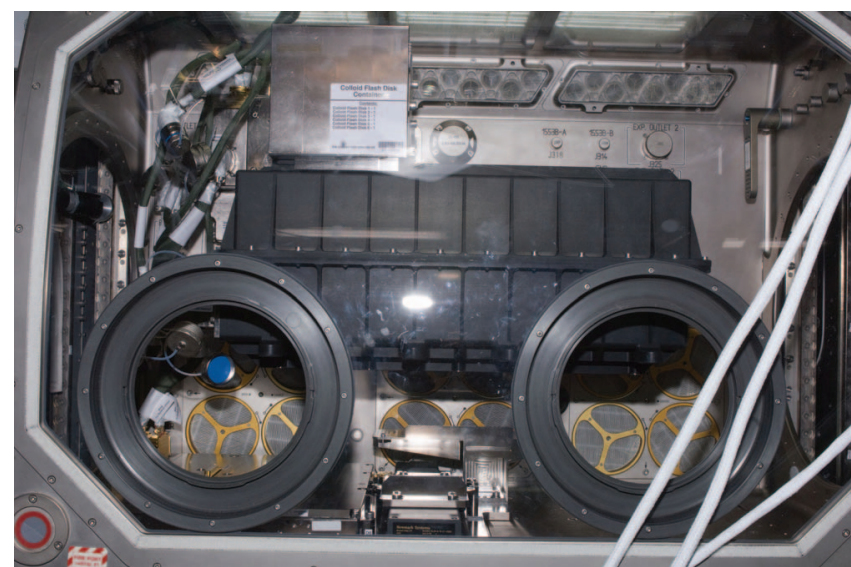

FIG. 3. Photo of the COLLOID module hosted inside the Microgravity Science Glovebox that hosts all the ancillary systems for power and data communication and storage, instrument control and on board image processing.

containment to fulfil safety requirements for instruments that are operated in manned spacecrafts like the ISS. Cells are individually temperature controlled by means of Kapton resistive heaters that were preferred over Peltier elements as the experiment requires the temperature of the cell to be increased only. Cells are cooled by means of conduction to housing of the COLLOID container, then dissipation into the air by forced convection provided by four fans mounted on the external side of the NFS module container. Since the thermal performance of the system could not be ultimately verified on ground because the lack of gravity-induced convection makes thermal equilibration much slower in space, a detailed analysis to simulate the thermal behaviour in microgravity was performed, predicting an average cooling rate of $5 \times 10^{-2} \mathrm{~K} / \mathrm{min}$ inside the box. Cells were also equipped with a Teflon-coated magnetic stirrer to (re)disperse the particles in the cell volume at the beginning of each experiment run. The stirrer was activated by moving the cell array through an array of magnets with alternating polarity.

The NFS module is accompanied by other SODI ancillary elements (see Fig. 3). The Facility Control Unit (FCU) hosts the drivers that control all SODI functions (e.g., motors, laser diode, temperature control system, etc.). The Image Processing Unit (IPU) hosts a single-board computer and a set of exchangeable solid state hard drives for images and data storage. The IPU is connected via serial data link to the Microgravity Science Glovebox (MSG), through which communication with ground control is established. In addition, an Ethernet link between the IPU and the MSG laptop computer allows science data to be retrieved to ground for intermediate analysis by the science teams. The IPU is connected to the main $(120 \mathrm{~V})$ and secondary $(28 \mathrm{~V})$ lines provided by the MSG, that supply the $230 \mathrm{~W}$ consumed by the SODI during operations.

Due to the limited availability of astronaut time on board the ISS, the instrument has been conceived to minimise crew interventions during operations. The system allows the execution of the experiments in full automatic mode, following a script loaded on the SODI computer. Ground operators can stop or modify the sequence by uploading a new script or run the experiment in manual mode, by sending a series of commands from ground. As a result, astronauts are only needed for a minimum number of operations, that is, the installation/de-installation of the instrument and the exchange of flash drives.

To be able to follow the experiment from ground, the instrument transmits telemetry and housekeeping data to ground, including key scientific quantities computed by the single board computer of the IPU. The image acquisition scheme adopted for the space experiment is based on the acquisition of batches of NFS images (typically composed of 100 frames). For each and every image, the mean intensity and normalized variance of the intensity (NVI) (see Appendix C) is computed, while the structure factor $\mathrm{S}(\mathrm{q})$ is computed over the last 10 NFS images of a batch, to limit the utilisation of the CPU. These quantities, along with temperature readouts from the cells, are sent to ground in quasi real-time (i.e., with a variable delay dictated by periods of loss of signal from the ISS when not orbiting in the range of any ground stations). As customary in the development of space instrumentation, a flight model (FM) and an engineering model (EM) were built, the latter essentially a replica of the flight instrument available for ground tests and validation of software scripts.

\section{COLLOIDAL SAMPLE}

The SODI-COLLOID module was used to study nanoparticles that experience a short-range attractive force due to critical Casimir forces, as predicted by Fisher and De Gennes $^{6}$ in 1978 . When stabilised particles are suspended in a liquid medium with large (e.g., larger than the Debye screening length in the case of charge stabilised particles) fluctuations of density, they experience a net attractive force due to the confinement of density fluctuations between pairs of particles. Despite being predicted more than 30 years ago, the first experimental observation of critical Casimir forces was reported in single-particle measurements of 2008. ${ }^{7}$ More recently, it was shown that critical Casimir forces are akin to the well-known depletion interaction. ${ }^{8}$ The experiments reported here aim at studying aggregation of colloidal particles as induced by critical Casimir forces. The main objective of the study is to relate the fractal dimension of the aggregates to the strength of the attractive Casimir force, in turn dependent by the temperature at which aggregation is taking place. This provides a new way to guide the aggregation with active control over the particle pair potential, alternatively to traditional aggregation mechanisms that rely on the screening of surface, ${ }^{9}$ in view of its potential utilisation for the growth of nanostructured materials. The very low strength of Casimir forces results in the creation of very fragile aggregates that can be modified or even disrupted by any macroscopic flow. As a result, these experiments need to be performed in absence of any convection or sedimentation. While the latter condition can be reached on ground through matching the density of particles with that of the fluid (even though for a narrow temperature range), convective flows are always present due to parasitic lateral temperature gradients. As such, the only way to obtain a 
flow-free environment in presence of temperature and concentration gradients and over a temperature range of the order of a few tens of degrees Celsius is to perform the experiment in microgravity condition. The system chosen is a "binary" mixture of 3-methyl pyridine (3MP) and water/heavy water with a $3 \mathrm{MP}$ weight fraction of $\mathrm{X}_{3 \mathrm{MP}}=0.39$ and a $\mathrm{D}_{2} \mathrm{O} / \mathrm{H}_{2} \mathrm{O}$ weight fraction of $\mathrm{X}_{\mathrm{hw}}=0.63$. The $3 \mathrm{MP} / \mathrm{D}_{2} \mathrm{O} / \mathrm{H}_{2} \mathrm{O}$ mixture has a lower consolution point at $49^{\circ} \mathrm{C} .{ }^{10}$ Teflon ${ }^{\circledR}$ colloidal particles of size $200 \mathrm{~nm}$ (radius) were suspended in the $3 \mathrm{MP} / \mathrm{D}_{2} \mathrm{O} / \mathrm{H}_{2} \mathrm{O}$ mixture at a volume fraction of $\sim 10^{-4}$, each cell with a different concentration of salt to provide different screening of the electrostatic repulsion induced by the surface charges. Previous experimental studies on this colloidal system ${ }^{1}$ show that critical Casimir-induced aggregation takes place at a temperature $\mathrm{T}_{\text {agg }}$ that is typically a few ${ }^{\circ} \mathrm{C}$ below (depending on salt concentration, $\mathrm{X}_{3 \mathrm{MP}}$ and $\mathrm{X}_{\mathrm{hw}}$ ) the critical consolution point of the mixture. The measurement of $T_{a g g}$, the study of the growth and morphology of the aggregates in reduced gravity conditions is the subject of the SODI-COLLOID experiment.

The SODI-COLLOID module was launched to the ISS on Soyuz Progress M-07M and activated on 14th of September 2010 for a first set of experiment that lasted until 11 th of October. NFS images and science data were recorded on hard disks and sent to ground with the final Space Shuttle Discovery flight, STS-133. The instrument was then stowed until it was installed again on the 17th of October 2011 for a second mission that lasted two weeks. At the time of writing, results of the static and dynamic analysis of the NFS images from the first mission have been submitted for publication, ${ }^{11}$ while the images from the second mission were delivered to the science team in the first half of 2012. We will here present a summary of the scientific data produced by the instrument.

\section{ANALYSIS OF NFS DATA}

\section{A. Static NFS measurements}

The simplest and most straightforward application of NFS is the measurement of the form factor $S(q)$ of the sample. Here, $q=2 k \sin \left(\frac{\theta}{2}\right)$ is the wave vector, $k$ the wave number, and $\theta$ the scattering angle. The measurement of $S(q)$ is achieved through the processing of a sequence of NFS images, where we indicate the intensity values recorded at position $(x, y)$ over the sensor plane for the $i$ th frame with $I_{i}(x, y)$. The procedure is as follows:

(a) An array of normalized differences between couples of frames at a fixed time distance $(i-j)$ is generated

$$
d_{i}(x, y)=\frac{I_{i}(x, y)-I_{j}(x, y)}{I_{i}(x, y)+I_{j}(x, y)} .
$$

Working with image differences allows eliminating static optical background (stray light) from the signal. However, time dependent spurious contributions (e.g., vibration) are not removed.

(b) The two-dimensional (2D) power spectrum (PS) of each difference is calculated using

$$
J_{i}\left(q_{x}, q_{y}\right)=\left|\mathcal{F}\left[d_{i}(x, y)\right]\right|^{2},
$$

where $\mathcal{F}$ indicates the Fourier transform. As mentioned above, the power spectrum of image differences does not contain contributions from static stray light, but it is affected by sensor noise (e.g., shot noise) that cannot be eliminated by image subtraction. It is however still possible to account for the sensor noise by subtracting the two-dimensional power spectrum $J_{i}{ }^{e}\left(q_{x}, q_{y}\right)$ of an "empty" cell, that is, a cell filled with filtered water, from $J_{i}\left(q_{x}, q_{y}\right)$. For the sample measured in the present paper, the contribution of $J_{i}{ }^{e}\left(q_{x}, q_{y}\right)$ proved to be very small and was therefore neglected.

(c) The power spectra are then averaged

$$
J\left(q_{x}, q_{y}\right)=\left\langle J_{i}\left(q_{x}, q_{y}\right)\right\rangle_{i}
$$

to obtain the power spectral density measured by the instrument as a function of the two-dimensional wave vector $\left(q_{x}, q_{y}\right)$.

(d) The power spectrum $J\left(q_{x}, q_{y}\right)$ is azimuthally averaged

$$
J(q)=\left\langle J\left(q_{x}, q_{y}\right)\right\rangle_{\theta} .
$$

(e) The azimuthally averaged power spectrum $J(q)$ is finally normalised by the Modulation Transfer Function (MTF) of the instrument $T(q)$, giving the form factor $S(q)$

$$
S(q)=\frac{J(q)}{T(q)} .
$$

The MTF has to be determined through a dedicated calibration procedure, described in Appendix A.

\section{B. Dynamic NFS measurements}

Brownian motions of the scatterers within the sample cause the speckle field to fluctuate in time. As shown in Ref. 12, these fluctuations can be exploited to recover statistical information about the diffusion of the aggregates, thus reproducing the traditional DLS method. Because of the heterodyne conditions, the intensity fluctuations are proportional to the real part of the scattered field. Therefore, time constants of the field-field correlation function of the system are measured, differently from traditional DLS where intensityintensity correlations are obtained. This represents an advantage of the NFS method, since different contributions to the correlation function actually add as fields.

Let us consider a pair of intensity distributions recorded at times $t$ and $t+\tau$, respectively. We consider now the explicit dependence of the acquisition time lag $\tau$ on the form factor calculated following the procedure outlined in Sec. V A (without averaging over time as in point c). Following Ref. 12 we can write

$$
S_{t}(q, \tau)=4 S_{t}(q)-4 \operatorname{Re}\left\{E(q, \tau) E^{*}(q, t+\tau)\right\},
$$

where $S_{t}(q)=S_{i}(q, \tau \rightarrow \infty)$ is the static power spectrum of the sample calculated at time $t$ in the limit of large $\tau$, while the second term is the field-field correlation function $G_{E}(q, \tau)$ $=\operatorname{Re}\left\{E(q, \tau) E^{*}(q, t+\tau)\right\} . S_{t}(q, \tau)$ thus provides the fieldfield correlation function at time $\mathrm{t}$ for a fixed $\tau$ over the entire accessible $q$-vector range.

To measure the time correlation functions, power spectra of the normalized differences for several delay times are 
computed. By evaluating $S_{t}(q, \tau)$ for various $\tau$, we create a sequence of field-field correlation functions. The time correlation function $S_{t}\left(q^{*}, \tau\right)$ can then be built for a fixed $q^{*}$.

On ground, sedimentation and convection can disturb and, depending on their strength, completely disrupt the diffusive motion of particles under observation. However, being collective motions of the sample over the field of view, they can be easily measured from $S_{i j}{ }^{13,14}$ For the COLLOID samples on ground, these motions drastically affect the speckle fields, so that the dynamic method could not be applied. By contrast, in microgravity sedimentation and convection are suppressed and the functional form of $S_{i}\left(q^{*}, t\right)$ is determined solely by diffusion.

As it is well known (see, for example, Ref. 15), the fieldfield correlation function of a system of mono-disperse noninteracting particles is described by a decreasing exponential, with a time constant determined by the Stokes-Einstein diffusion coefficient, D, according to

$$
\begin{aligned}
S_{t}\left(q^{*}, \tau\right) & =A\left(q^{*}\right) e^{-\frac{\tau}{\tau_{D}}}, \\
\tau_{D} & =\frac{1}{D q^{2}} .
\end{aligned}
$$

The exponential characteristic times, and therefore the hydrodynamic radii $R_{h}$, can be easily fitted to data over the available $q$ range, that for this analysis effectively ranged from 0.7 to $2 \mu \mathrm{m}^{-1}$. Having access to a whole wave vector range at once presents an important advantage in terms of statistics that partially overcomes the relatively limited time range accessed by COLLOID. Even if the time lag spans just a couple of decades, from $1 \mathrm{~s}$ to $100 \mathrm{~s}$, changing $q$ by a factor 3 allows extending the time lag by almost an additional decade.

\section{MEASUREMENT RESULTS}

\section{A. Normalized variance of the intensity}

Before the actual measurements of the growth of aggregates, we first determined the temperature $T_{\text {agg }}$, at which aggregation starts. This temperature depends on the amount of added salt, and has therefore to be measured for each cell separately. To determine $\mathrm{T}_{\text {agg }}$, we continuously raised the temperature and monitored the NVI. A change of the NVI indicates a change of the number density of scatterers or their cross section, and provides a good measure of the growth of aggregates. The temporal evolution of the NVI for one of the space samples is shown in Fig. 4. The figure shows data taken over approximately $18 \mathrm{~h}$, during which the temperature of the system was progressively increased. The strong increase in the NVI demarcates the start of the aggregation of the particles. Thus, in this particular example, $\mathrm{T}_{\mathrm{agg}}=44.2^{\circ} \mathrm{C}$ This sequence was performed as a first step for all samples, in order to locate $\mathrm{T}_{\text {agg }}$. The aggregation temperatures for all cells were then used in subsequent runs, in which the samples were brought from initial ambient temperature to the respective $T_{\text {agg }}$. As soon as $T_{\text {agg }}$ was reached within $\pm 0.01 \mathrm{~K}$, a set of 100 NFS images was acquired. The NVI, as clearly visible in Figure 4 is an excellent indicator of the formation of aggregates, being linked to the forward scattering $S(0)$ (see

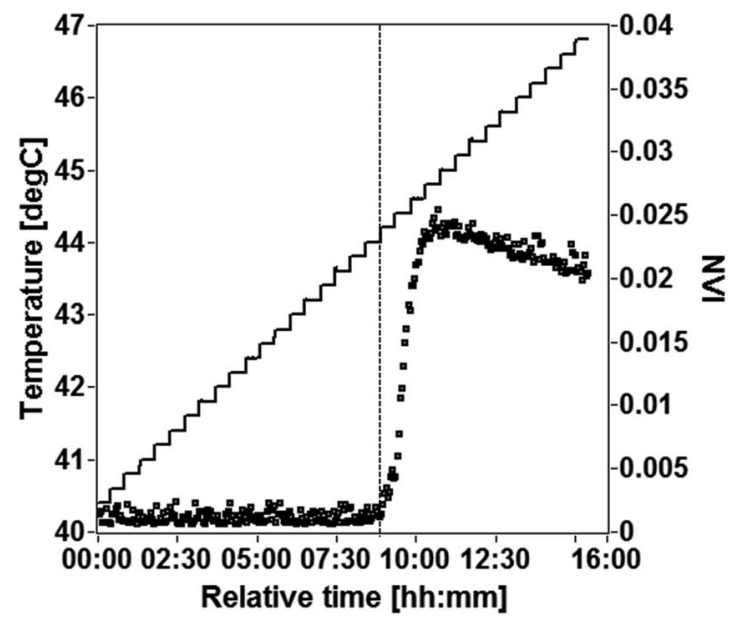

FIG. 4. Time evolution of the NVI for cell 2 during the first experimental run. Temperature inside the cell was progressively raised in order to locate the aggregation temperature $T_{\text {agg }}$. The vertical dotted line indicates the measured aggregation temperature, $\mathrm{T}_{\mathrm{agg}}=44.2^{\circ} \mathrm{C}$.

Eq. (C10)) and therefore very sensitive to the size of the scatterers. In fact, the formation of aggregates boosts the variance by a factor of 10 , from a background value of $2 \times 10^{-3}$ for all of the samples under observation. It is worth noting that ground tests performed on samples that were the replica of the ones used in space resulted in even higher values of NVI after aggregation, reaching values of $10^{-1}$ and starting from comparable background values. Since the NVI is linearly proportional to the number of scattering elements $\mathrm{N}$ that are present in the scattering volume this could be explained by non-complete suspension of the particles that were left sedimenting during the 5 months between the filling on ground and the activation of the experiment in space, resulting in fewer particles in the cell volume. In addition, sedimentation is exacerbated by the launch phase, when the sample is exposed to accelerations up to $3 \mathrm{~g}$ for several minutes.

\section{B. Static NFS}

Figure 5 shows the form factor of the sample as measured during the space experiment. The top panel shows the measured power spectrum $J(q)$. The form factor $S(q)$ as defined in (5) is shown in the bottom panel. The correction by the MTF changes the shape of the power spectrum slightly. Furthermore, one can notice the presence of oscillations in both the curves at low $q$ (i.e., $q<2 \times 10^{-1} \mu \mathrm{m}^{-1}$ ), that are not accounted for by the MTF. Such oscillations are due to a combination of two phenomena described in Appendix B, and can mask $S(q)$ at low wave vector. It is however possible to recover the signal at low wave vectors through a procedure described in Appendix B.

The measured form factor is characteristic for finite, fractal aggregates. To show this, we use the well-known FisherBurford expression ${ }^{16}$ that links the key geometrical quantities of the aggregate to the form factor

$$
S(q) \propto \frac{1}{\left[1+2\left(q R_{g}\right)^{2} / 3 d_{f}\right]^{d_{f} / 2}},
$$



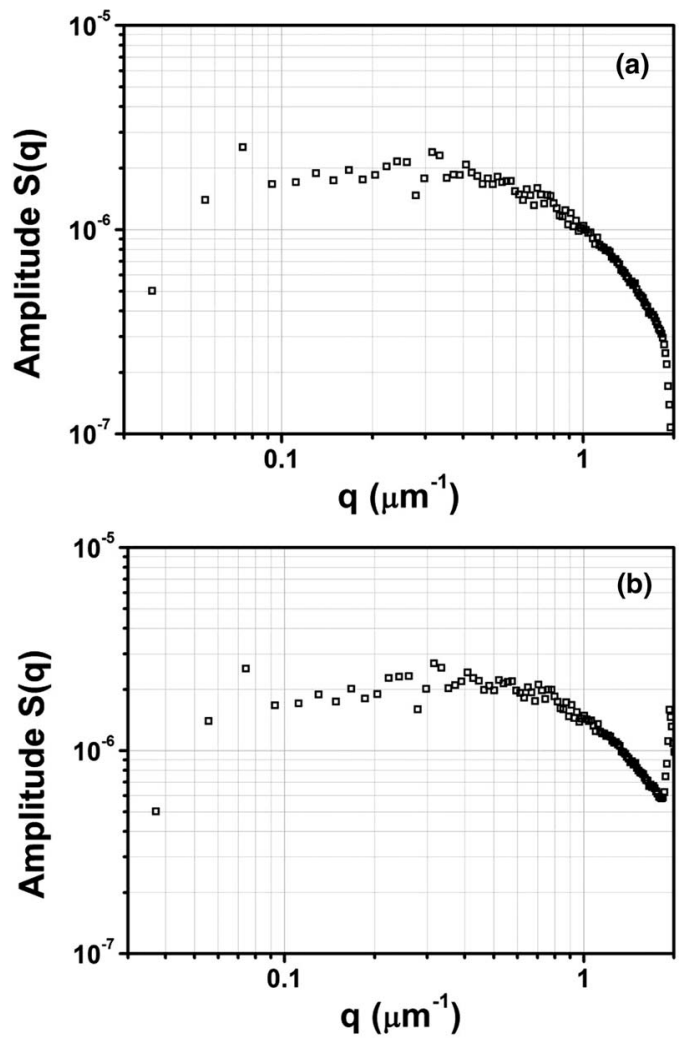

FIG. 5. (a) An example of a raw PS J(q); (b) the MTF corresponding S(q) corrected for the MTF T(q).

where $R_{g}$ is the gyration radius of the aggregates and $d_{f}$ the fractal dimension.

It is therefore possible to measure the growth of aggregates by evaluating $S(q)$ as a function of time. Figure 6 shows a set of $S(q)$ curves acquired during aggregation at different times together with best fits using Eq. (8). One can notice that the overall signal grows as a function of time, indicating that aggregates are growing, progressively increasing the amount of scattered light. The agreement between the data and the fits is generally quite good, except for points below $0.3 \mu \mathrm{m}^{-1}$

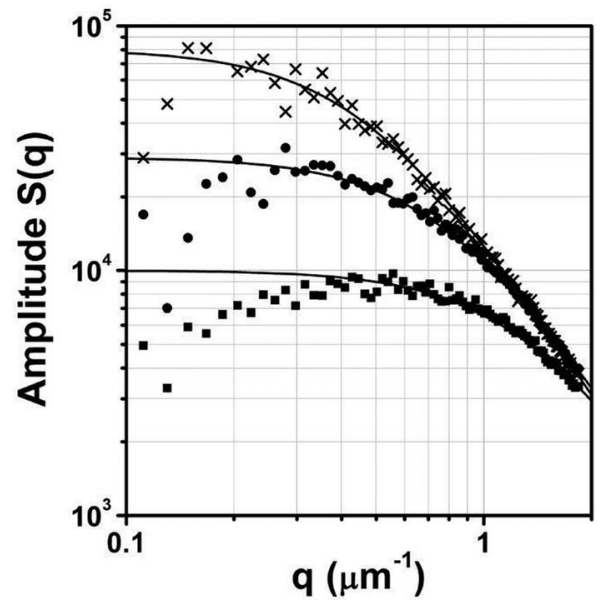

FIG. 6. Sequence of $\mathrm{S}(\mathrm{q})$ curves acquired during aggregation, at $\mathrm{t}=200 \mathrm{~s}$ (squares), $\mathrm{t}=700 \mathrm{~s}$ (circles) and $\mathrm{t}=2100 \mathrm{~s}$ (crosses), time $\mathrm{t}=0$ being the beginning of aggregation. Solid curves are data fit with the corresponding Fisher-Burford curves.
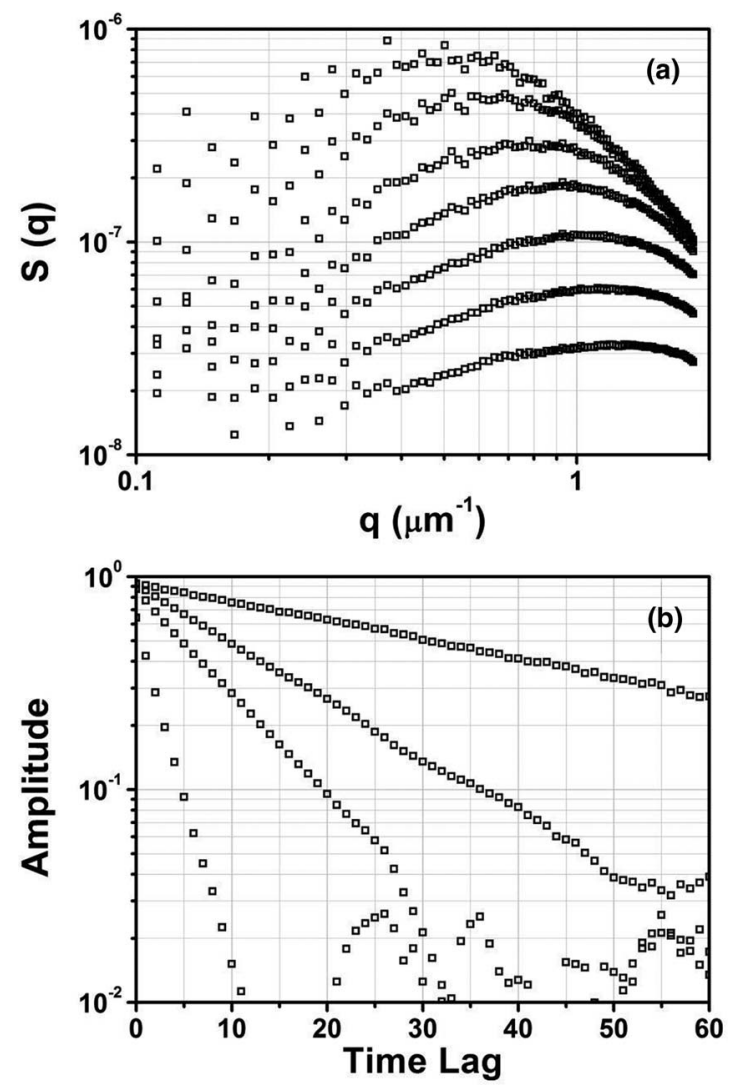

FIG. 7. (a) $S(q)$ as a function of the lag time $(1,2,4,8,16,32,64 \mathrm{~s}$, from the bottom as indicated by the arrow); (b) example of correlation functions obtained from the $\mathrm{S}(\mathrm{q})$ for $\mathrm{q}=0.3,0.7,1,1.75 \mu \mathrm{m}^{-1}$ (from above).

where a depression in the power spectral density is present due to the interactions between the scatterers. This allows not only to obtain the average gyration radius $R_{g}$ of the aggregates as a function of time but also the fractal dimension from the slope at high wavelengths, where $S(q) \sim q^{-d_{f}}$. The best fit to the data in Fig. 6 gives $R_{g}=1.35,2.3$, and $2.9 \mu \mathrm{m}$ and $\mathrm{d}_{\mathrm{f}}=2.2$.

\section{Dynamic NFS}

To evaluate the dynamics, we show a typical time sequence of power spectra $S_{t}(q, \tau)$ obtained from COLLOID flight data in Fig. 7(a). We remind that $S_{i j}(q, \tau)$ is the form factor calculated from the difference of images recorded at times $t$ and $t+\tau$. As the time lag $\tau$ increases, the intensity distribution of the two images becomes progressively uncorrelated. As a result, the $S_{t}(q, \tau)$ grows approaching the "true" $S_{t}(q)$. The effect is $q$-dependent, as large wave vectors (i.e., spatially small objects) de-correlate faster due to the diffusive nature of the process (see Eq. (7)). We can therefore obtain the single correlation functions for the available wave vectors range, as shown in Figure 7(b) for a few q values.

Fitting to single exponentials has been performed, resulting in estimates for the characteristic decorrelation times. For the correlation functions presented in Fig. 7(b), the fitting times are $2.5 \mathrm{~s}, 9 \mathrm{~s}, 16 \mathrm{~s}, 46 \mathrm{~s}$, respectively. Due to the limited range in lag times, no further information can be gathered, such as what can be obtained with the traditional 
cumulant methods for DLS. In addition, the determination of the Stokes-Einstein diffusion coefficients and therefore the hydrodynamic radii cannot be performed by a simple dynamic analysis. This is caused by the rotational degree of freedom of the aggregates, as rotations can lead to additional decorrelation unaccounted for by the Stokes-Einstein diffusion equation. As a matter of fact, for micrometer sized scatterers as measured in COLLOID, the correlation characteristic times for Brownian motions are close to the rotational diffusion times. Rotations then participate to depress the correlation functions, and must be kept into account for a precise data analysis as described in Sec. V D.

\section{Combining static and dynamic near field scattering}

The combination of simultaneous static and dynamic measurements allows to measure the hydrodynamic radii and to obtain insight into the internal structure of the aggregate. This method presents a unique advantage over the traditional light scattering technique, as one can in principle simultaneously determine the radius of gyration $R_{g}$ and the hydrodynamic radius $R_{h}$, thereby gathering information on the compactness of the aggregate. A proper analysis keeping into account the additional decorrelation coming from the rotations of aggregates can be achieved considering an effective diffusion coefficient $\mathrm{D}_{\text {eff }}$ as a function of $q^{17}$ that depends on both the hydrodynamic and the gyration radii of the scatterers. This approach allows to measure the ratio $\beta=R_{h} / R_{g}$ which is usually difficult to be assessed, and is connected to the internal structure of the aggregates. ${ }^{18}$ COLLOID proved to be an ideal instrument to exploit the analysis described in Ref. 17, overcoming the difficulties traditionally encountered thanks to the strong reduction of convection and sedimentation due to the microgravity conditions and the capability to recover both static and dynamic information starting from the same data.

Following the procedure described in Sec. V A, the static form factor $S(q)$ can be recovered, from which precise measurements for the gyration radius $R_{g}$ and the fractal dimension $d_{f}$ are obtained using the Fisher-Burford relation (8) (see, for example, Refs. 5 and 11). These parameters can be used to generate the expected dependence of the effective diffusion coefficient $D_{\text {eff }}(q)$ that, following Ref. 17 and considering the Fisher-Burford description for the static form factor $S\left(q, R_{g}\right)$, turns out to be related to the Stokes-Einstein diffusion coefficient $\mathrm{D}$ as follows:

$$
\frac{D_{e f f}}{D}=1+\frac{1}{2 \beta^{2}}\left[1-\frac{3 d_{f}}{3 d_{f}+2\left(q R_{g}\right)^{2}}\right] .
$$

The only free parameter here is the ratio $\beta=R_{h} / R_{g}$ and since $R_{g}$ is measured from the static structure factor, the value of $R_{h}$ can be determined by fitting to the measured q-dependent diffusion coefficient. As it appears from the previous equation, the ratio $D_{e f f} / D$ does depend on the product $q R_{g}$ only, thus defining a master curve that is independent of time.

Experimental measurements of the effective diffusion coefficient, and best fits using Eq. (9) are shown in Fig. 8. The two data sets represent measurements at two different temperatures, $\mathrm{T}_{\mathrm{agg}}$ and $\mathrm{T}_{\mathrm{agg}}+0.4 \mathrm{~K}$. The master curves fit the

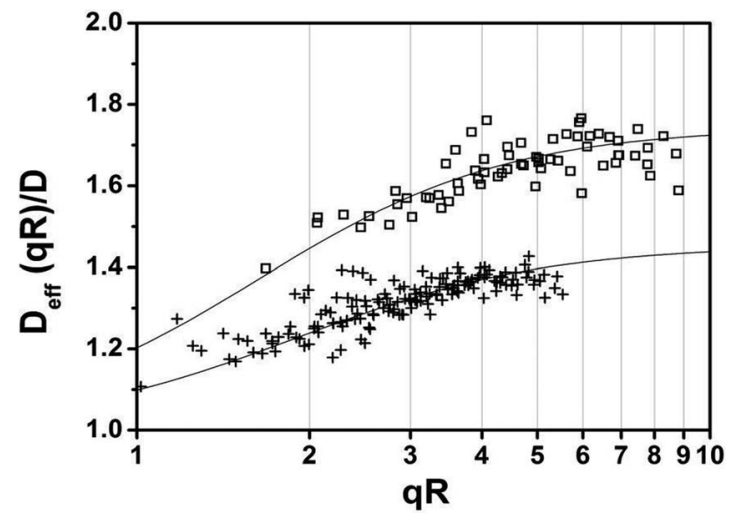

FIG. 8. Example of the master curves obtained from data at two different temperatures. The curves have been fitted to data by imposing $\beta=1.05$ (lower curve, $\mathrm{T}_{\mathrm{agg}}$ ) and $\beta=0.82$ (upper curve $\mathrm{T}_{\mathrm{agg}}+0.40 \mathrm{~K}$ ).

experimental data very well. The effect of the rotations is evident from the increasing $D_{\text {eff }} / D$ as a function of $q$. The higher $q$ region in the static form factors, and the corresponding correlation functions, are determined by the smaller structures in the scatterers. For highly structured scatterers, the rotation effects are larger, as it appears for the highest temperature data (upper curve). By contrast, the lower curve indicates that a more compact object is formed, in agreement with the higher fractal dimension measured from the static form factors. ${ }^{11}$ In this way, the measure of $\beta$ provides additional information about different structures within the aggregates.

\section{CONCLUSION}

In this paper, we have presented the SODI-COLLOID instrument, a compact near field scattering machine built for microgravity experiments. This rather novel technique has been preferred over more traditional ones due to its very simple setup and data acquisition scheme. The instrument has been launched to the ISS in 2009 to measure aggregation of colloids induced by critical Casimir forces. Selected results from the analysis of the first space experiment (2010) were presented. Notwithstanding its simplicity, NFS is a very versatile technique that can combine static and dynamic light scattering measurements. In particular, it can measure independently both the hydrodynamic $R_{h}$ and gyration radius $R_{g}$ of the aggregates, thus giving information on their internal structure. The potential of NFS is best exploited in reduced gravity conditions, where any buoyancy driven large scale flow is strongly suppressed. This is crucial for the dynamic analysis of data, which is based on subtraction of NFS images acquired at different times. Without the contribution of sedimentation or convection, Brownian motion, and rotation of the growing aggregates remain the only responsible for the decorrelation of NFS images, allowing a precise and straightforward measurement of the diffusion coefficient. This considered, NFS proved to be the ideal tool for static and dynamic measurements of aggregation of nanoparticles in space. 


\section{APPENDIX A: CALIBRATION PROCEDURE}

As mentioned in Sec. V, the experimental data have to be corrected for the MTF of the light scattering instrument. This MTF was determined for both the FM and the EM. In principle, since the output of NFS is an image, the full calibration procedure requires the characterisation of the twodimensional MTF. However, since the final data are obtained through an azimuthal average of the power spectra of such NFS images, it is enough to measure the azimuthal average of the transfer function. This greatly simplifies the calibration procedure.

The microscope objectives used, produce magnifications slightly different from the reported ones, in part due to deviations from a perfect collimation. The actual magnification of the overall system was determined by using a static speckle pattern generated by a simple ground glass mounted upon a micrometric translation stage. By using the properties of the near field speckles, ${ }^{19}$ we calibrated the magnification $\mathrm{M}$ on the basis of known displacements. The pixel size, $p$, is fixed by the choice of the camera, or equivalently the sensor size, $L=N p$, where $N \times N$ is the total number of pixels. By determining the actual magnification of the objective used, the pixel size in Fourier space can be calculated by means of $q_{\min }$ $=2 \pi M / L$. This was determined separately for the FM and the EM.

In order to determine the MTF, a calibration sample was used containing a suspension of polystyrene spheres of $2 \mu \mathrm{m}$ in diameter in 50\% water, $50 \%$ deuterated water, and an "empty" sample containing only the water. By using a mixture of water and heavy water, the density of the solvent could be matched to the density of the particles, thereby greatly reducing their sedimentation. The power spectrum of the particles as calculated from Mie-theory remains almost flat over the entire COLLOID wave vector range (Fig. 9 below).

In order to reduce the statistical errors to below $1 \%$ over the entire wave vector range, a set of 10000 images was recorded with an acquisition frequency of $1 \mathrm{~Hz}$. We follow the same scheme as adopted for the static analysis (see Sec. V) in order to obtain power spectra for both the calibration sample and the "empty" sample, averaged over the entire

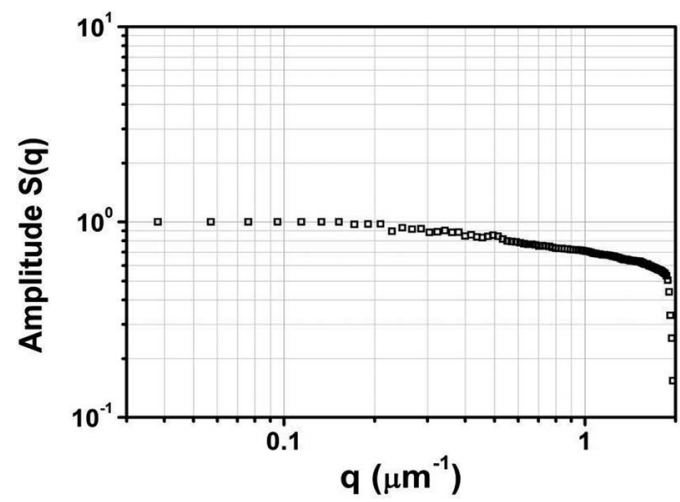

FIG. 9. The modulation transfer function for the COLLOID Flight Model (open squares). The continuous line represents the differential cross section (arbitrary units) of polystyrene spheres $2 \mu \mathrm{m}$ in diameter suspended in water for a wavelength $\lambda=935 \mathrm{~nm}$, as a function of the transferred wave vector as obtained from Mie scattering calculations. data set. The resulting average PS of the "empty" sample is then subtracted from the average PS of the calibration sample in order to remove noise generated by the camera (shotnoise). The result provides a measurement of the power spectral density measured by the instrument as a function of the two-dimensional wave vector $\left(q_{x}, q_{y}\right)$. After azimuthally averaging, the resulting PS, $J(q)$, is finally divided by the expected form factor $I(q)$ in order to find the MTF of the instrument. The expected form factor $I(q)$ for the calibration sample is obtained through accurate Mie calculations for the differential cross section as a function of the transferred wave vector $\mathrm{q}$ at a wavelength of $\lambda=935 \mathrm{~nm}$

$$
\operatorname{MTF}(q)=\frac{J(q)}{I(q)} .
$$

The resulting MTF for the COLLOID FM is shown in Fig. 9 which is used to correct the raw experimental data. The MTF shows that the system is capable of providing information up to a wave vector $\mathrm{q}=1.9 \mu \mathrm{m}^{-1}$, corresponding to the nominal numerical aperture of the microscope objective.

\section{APPENDIX B: MODIFIED TALBOT EFFECT}

At wave vectors lower than $0.5 \mu \mathrm{m}^{-1}$, a small oscillatory behaviour is present even after calibration, as can be seen in Fig. 5(b). This is due to the combination of two different physical effects that must be taken into account. One is the so called Talbot effect, which affects power spectra of images taken just downstream a sample with deep oscillatory modulations. ${ }^{20}$ This is due to scattering from 2D structures (Raman-Nath ${ }^{21}$ ) and disappears when the 3D Bragg scattering regime is reached. ${ }^{22}$ The second is a subtle effect which changes the oscillation phase with the size of the scatterers, therefore producing a scattering signal that is sample dependent. This effect was described and exploited in Ref. 23 and affects the measurements in two ways. As a result, the MTF determined from the calibration contains oscillations from the particles in the calibration sample which are obviously not present in the experimental system when the sample differs from the one used in the calibration. These oscillations are however sufficiently small and can be neglected, allowing the utilisation of the MTF to correct any experimental data.

Talbot oscillations, always present in the experimental data, can hide the "true" $S(q)$ at low q wave vectors (see Fig. 10(a)). In order to minimize the Talbot oscillations as much as possible, the cell was thick enough to meet the 3D Bragg scattering conditions.

Furthermore, it is possible to compensate for these oscillations by modelling their behaviour in the actual working conditions simply by using the (unknown) size of the scatterers as a fitting parameter. A simple explanation of the Talbot effect can be found in Ref. 23 and references therein. In the case of a pure 2D scatterer (Raman-Nath conditions), it introduces a modulation in the PS given by

$$
T^{\prime}{ }_{2 D}(q)=\left[\sin ^{2}\left(\frac{q^{2} z}{2 k}\right)+\varphi\right],
$$

where $k=2 \pi / \lambda$ and $\varphi$ is the phase lag described in Ref. 23 . 

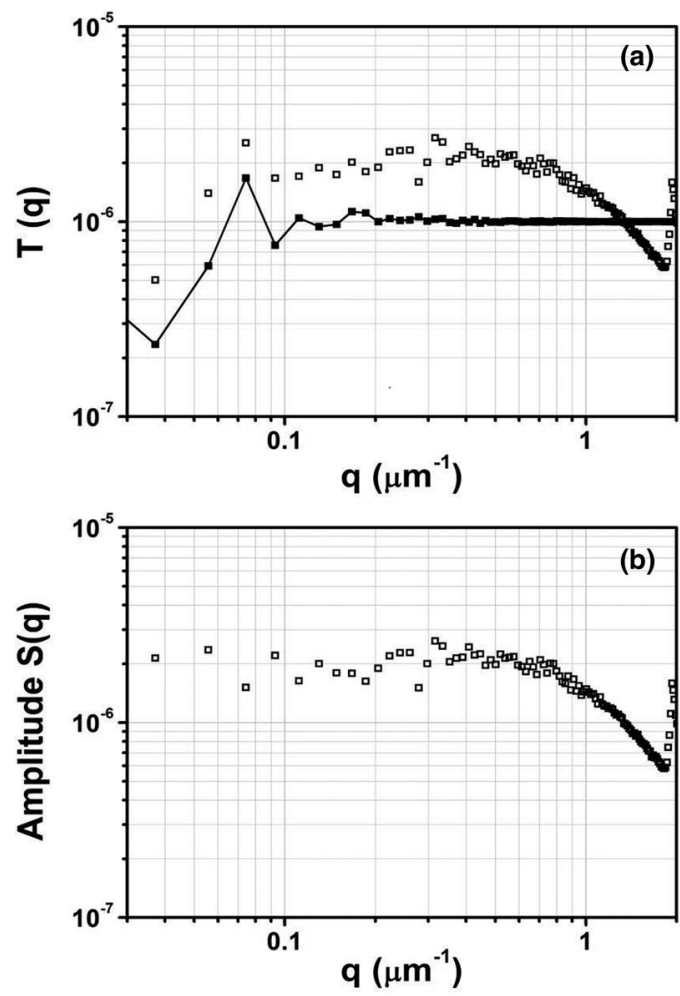

FIG. 10. (a) Measured PS compensated for the MTF (squares) and the fitting TTF multiplied by a factor $10^{4}$ (solid line); (b) the form factor S(q) after compensation for the modified Talbot effect.

Now we let the scattering system increase in depth up to a longitudinal thickness $L$. The Talbot effect changes since the contributions coming from different planes within the sample are sensed at position $z$ at different "effective" observation distances. By increasing $L$, the system continuously changes from a $2 \mathrm{D}$ to a $3 \mathrm{D}$ system. To put it more quantitatively, it is enough to integrate the Talbot Transfer Function (TTF) of Eq. (B1) from $z$ to $z+L$

$$
T^{\prime}(q)=1-\cos \left[\frac{q^{2}}{2 k}(2 z+L)+\varphi\right] \operatorname{sinc}\left(\frac{q^{2} L}{2 k}\right),
$$

where we used the function sinc $x=\sin x / x$. The 3D Bragg effect is described by the last factor, which depends on $L$. This shows that the first node of the oscillations appears at $q$ equals

$$
q_{B}=\sqrt{\frac{2 \pi k}{L}} .
$$

The oscillations are also present at larger wave vectors, however, eventually vanishing a few cycles after $q_{B}$. In Fig. 10(a), we show a plot of the oscillations as calculated for the specific conditions in COLLOID.

This result does not include the tapering of the oscillations due to the geometrical effect described in Ref. 23. This effect can however be neglected since the distance $z$ is comparable to the thickness $L$ in which case the 2D-3D transition effect is dominant. Notice that Eq. (B2), contains a substantial simplification. The thickness must include the sample refractive index and the optical path through the cell walls resulting in slightly more complicated calculations. This complication can be avoided however, by introducing an effective cell thick- ness at the working wavelength. That is, one can first fit the main (Talbot) oscillations by changing $z$ and $\varphi$, and finally the amplitude of the Talbot oscillations. This amplitude can be affected by some spurious effects, however, this reduction is only approximately $10 \%$. The analytical function is then used to divide the PS to produce the form factor $S(q)$ of the sample. An example is shown in Fig. 10, where in (a) we show the PS together with the fitted $T^{\prime}(q)$, and in (b) the resulting form factor.

\section{APPENDIX C: THE SIGNAL VARIANCE AND THE COMPLEX SCATTERED AMPLITUDE}

We have shown that, once the MTF and the TTF (see Eq. (B2)) are known and characterised, the form factor $S(q)$ can be obtained following a procedure outlined in Sec. V. Besides $S(q)$, NFS allows to extract information about the amplitude of the scattering field through the variance of the NFS images, thanks to the self-reference condition. This is a very valuable feature for a space-borne instrument as it allows monitoring of the progression of the experiment by sending a quantity (the variance of images) that can be easily computed by the on-board software to mission control centres, which is less demanding than images in terms of bandwidth occupation. In this section, we show how to determine the adimensional amplitude of the scattered field, $S(0)$, thus obtaining information about the complex field of the scattered waves.

Let $A_{S}$ be the amplitude of the emerging wavefront, which is much smaller than the transmitted field amplitude, $A_{T}$, to guarantee the heterodyne condition. At position $r=(x, y, z)$ on the sensor, let $A_{S}$ be the superposition of $N$ spherical scattered waves with amplitudes given by

$$
a_{j}(x, y)=|S(0)| \frac{A_{j}}{k_{0}\left|r-r_{j}\right|}
$$

and random phases $\varphi_{j}(x, y)$. Here, $A_{j}=A\left(r_{j}\right)$ is the amplitude of the field impinging onto the $j$ th scatterer, placed at position $r_{j}=\left(x_{j}, y_{j}, z_{j}\right)$. The interference with the transmitted field produces a speckled intensity distribution given by

$$
I(x, y)=A_{T}^{2}+2 A_{T} \operatorname{Re}\left[\sum_{j=1}^{N} a_{j} \exp \left(i \varphi_{j}\right)\right] .
$$

This speckle field is characterized by a first order intensity distribution described by a modified Rician distribution, that under the heterodyne condition reduces to a Gaussian function with average $A_{T}{ }^{2}$ and a variance $V_{I}$ given by ${ }^{24}$

$$
V_{I}=4 \sigma^{2}\left(A_{T}^{2}+\sigma^{2}\right) \sim 4 \sigma^{2} A_{T}^{2},
$$

where $2 \sigma^{2}=\sum_{j=1}^{N} a_{j}{ }^{2}=N\left\langle a_{j}{ }^{2}\right\rangle$. As a consequence, we get the following expression for the variance of the intensity:

$$
V_{I}=2|S(0)|^{2} \frac{A_{T}^{2}}{k_{0}^{2} z^{2}} \sum_{j=1}^{N} A_{j}^{2},
$$

where the paraxial approximation $\left|r-r_{j}\right|=z$ has been used for any $j$.

In the experimental setup, the $\mathrm{N}$ particles are illuminated by a beam with a Gaussian intensity profile. To take this into account, we assume that the amplitude of the scattered waves 
depends only on the distance from the optical axis, $r=z \theta$, so that any distance $r$ is associated with a value $I(r)$ for the intensity of the incoming beam

$$
|A(r)|^{2}=I(r)=I_{m} \exp \left(-\frac{r^{2}}{r_{0}^{2}}\right),
$$

where $r_{0}$ is the distance at which the beam intensity drops to a value $1 / e$ of the maximum, $I_{m}$. This assumption is well satisfied in COLLOID, since the magnification of the system is a factor 20 approximately. In this case, the effective observation region has an extension around the optical axis that is much smaller than the illuminated region. We shall concentrate on the simple case of a collection of $n$ identical scattering centres per unit volume. For a given annular region of the sample between a distance $r$ and $r+d r$ from the optical axis, the variance of the speckle field is given by

$$
d V_{I}=2 n 2 \pi r d r L|S(0)|^{2} \frac{A_{T}^{2}}{k_{0}^{2} z^{2}} I_{m} \exp \left(-\frac{r^{2}}{r_{0}^{2}}\right),
$$

where $L$ is the sample thickness. The integration is extended from $r=0$ up to a distance $r=r_{\text {max }}$ imposed by the geometry of the cell. The expression for the variance then becomes

$$
V_{I}=4 n \pi L|S(0)|^{2} \frac{A_{T}^{2}}{k_{0}^{2} z^{2}} I_{m} \int_{0}^{r_{\max }} \exp \left(-\frac{r^{2}}{r_{0}^{2}}\right) r d r .
$$

We now define the normalized intensity distribution as

$$
i(x, y)=\frac{I_{1}(x, y)-I_{2}(x, y)}{I_{1}(x, y)+I_{2}(x, y)}=\frac{1}{A_{T}} R e\left[\sum_{j=1}^{N} a_{j} \exp \left(i \varphi_{j}\right)\right]
$$

that produces a NVI given by

$$
N V I=\frac{V_{I}}{A_{T}^{2} A_{0}^{2}} .
$$

The result is independent of the incoming beam amplitude, $A(r)$ and the transmitted beam amplitude, $A_{T}$. When the extinction is very small, that is, $A_{T}=A_{m}$, the NVI reduces to

$$
N V I=\frac{V_{I}}{A_{T}^{4}}=4 n \pi L|S(0)|^{2} \frac{1}{k_{0}^{2} z^{2}} \int_{0}^{r_{\max }} \exp \left(-\frac{r^{2}}{r_{0}^{2}}\right) r d r .
$$

This result also shows that the NVI can be used to extract information about the adimensional scattering amplitude once the number concentration is known. This has been done to evaluate the effective concentration of monomers in the samples after the instrument launch and installation aboard the ISS.

${ }^{1}$ Reference Guide to the International Space Station, edited by G. Kitmacher (CreateSpace Independent Publishing Platform, 2010).

${ }^{2}$ D. Bonn, J. Otwinowski, S. Sacanna, H. Guo, G. Wegdam, and P. Schall, Phys. Rev. Lett. 103, 156101 (2009).

${ }^{3}$ M. Giglio, M. Carpineti, and A. Vailati, Phys. Rev. Lett. 85, 1416 (2000).

${ }^{4}$ D. Brogioli, A. Vailati, and M. Giglio, Appl. Phys. Lett. 81, 4109 (2002).

${ }^{5}$ F. Ferri, D. Magatti, D. Pescini, M. A. C. Potenza, and M. Giglio, Phys. Rev. E 70, 041405 (2004).

${ }^{6}$ M. E. Fisher and P.-G. de Gennes, Acad. Sci., Paris, C. R. 287, 207 (1978).

${ }^{7}$ C. Hertlein, L. Helden, A. Gambassi, S. Dietrich, and C. Bechinger, Nature (London) 451, 172 (2008).

${ }^{8}$ S. Buzzaccaro, J. Colombo, A. Parola, and R. Piazza, Phys. Rev. Lett. 105, 198301 (2010).

${ }^{9}$ E. J. W. Verwey and J. Th. G. Overbeek, Theory of Stability of Lyophobic Colloids (Elsevier, Amsterdam, 1948).

${ }^{10}$ B. V. Prafulla, T. Narayanan, and A. Kumar, Phys. Rev. A 46, 7456 (1992).

${ }^{11}$ S. J. Veen, O. Antoniuk, B. Weber, M. Potenza, S. Mazzoni, P. Schall, and G. H. Wegdam, Phys. Rev. Lett. 109, 248302 (2012).

${ }^{12}$ D. Magatti, M. D. Alaimo, M. A. C. Potenza, and F. Ferri, Appl. Phys. Lett. 92, 241101 (2008)

${ }^{13}$ M. A. C. Potenza, M. D. Alaimo, D. Pescini, D. Magatti, F. Ferri, and M. Giglio, Opt. Lasers Eng. 44, 722 (2006).

${ }^{14}$ M. D. Alaimo, M. A. C. Potenza, D. Magatti, and F. Ferri, J. Appl. Phys. 102, 073113 (2007).

${ }^{15} \mathrm{~J}$. W. Goodman, in Laser Speckle and Related Phenomena, edited by J. C. Dainty (Springer, Berlin, 1975).

${ }^{16}$ M. E. Fisher and R. J. Burford, Phys. Rev. 156, 583 (1967).

${ }^{17}$ M. Y. Lin, H. M. Lindsay, D. A. Weitz, R. C. Ball, R. Klein, and P. Meakin, Proc. R. Soc. A 423, 71 (1989).

${ }^{18}$ P. Wiltzius, Phys. Rev. Lett. 58, 710 (1987).

${ }^{19}$ M. Alaimo, D. Magatti, F. Ferri, and M. A. C. Potenza, Appl. Phys. Lett. 88, 191101 (2006).

${ }^{20} \mathrm{~J}$. W. Goodman, Introduction to Fourier Optics (Roberts and Company Publishers, 2004).

${ }^{21}$ M. Born and E. Wolf, Principles of Optics (Cambridge University Press, 1999).

${ }^{22}$ M. Giglio, D. Brogioli, M. A. C. Potenza, and A. Vailati, Phys. Chem. Chem. Phys. 6, 1547 (2004).

${ }^{23}$ M. A. C. Potenza, K. P. V. Sabareesh, M. Carpineti, M. D. Alaimo, and M. Giglio, Phys. Rev. Lett. 105, 193901 (2010).

${ }^{24}$ J. W. Goodman, Statistical Optics (Wiley-Interscience, 2000). 